\title{
Preparation of Nanoscale TiO2 Dispersions in Biological Test Media for Toxicological Assessment
}

M. R. Wiesner Duke University Department of Civil and Environmental Engineering

Durham, NC 27708

http://dx.doi.org/10.6028/NIST.SP.1200-4
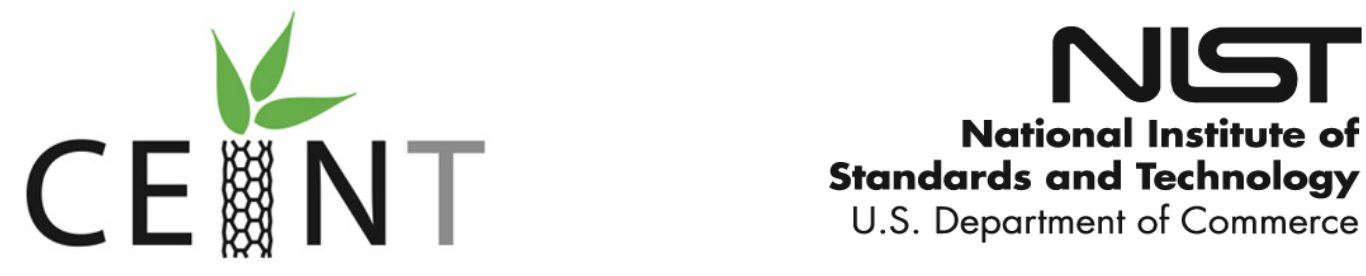

National Institute of Standards and Technology U.S. Department of Commerce 


\section{Preparation of Nanoscale $\mathrm{TiO}_{2}$ Dispersions in Biological Test Media for Toxicological Assessment}

Version 1.1

J. S. Taurozzi

V. A. Hackley

National Institute of Standards and Technology Material Measurement Laboratory Gaithersburg, MD 20899-8520

M. R. Wiesner Duke University Department of Civil and Environmental Engineering

Durham, NC 27708

http://dx.doi.org/10.6028/NIST.SP.1200-4

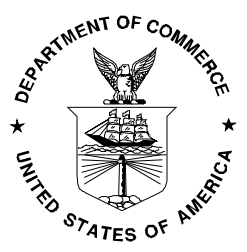

U.S. Department of Commerce John E. Bryson, Secretary

National Institute of Standards and Technology Patrick D. Gallagher, Under Secretary of Commerce for Standards and Technology and Director 
Certain commercial entities, equipment or materials may be identified in this document in order to describe an experimental procedure or concept adequately. Such identification is not intended to imply recommendation or endorsement by the National Institute of Standards and Technology, nor is it intended to imply that the entities, materials or equipment are necessarily the best available for the purpose.

National Institute of Standards and Technology Special Publication 1200-4 Natl. Inst. Stand. Technol. Spec. Publ. 1200-4, 13 pages (June 2012) 


\section{FOREWORD}

This special publication is one in a series of protocols resulting from a collaborative research agreement between the National Institute of Standards and Technology (NIST) and Duke University's Center for the Environmental Implications of Nanotechnology (CEINT). The original version of this protocol (Ver. 1.0) was first posted on the CEINT web site (http://ceint.duke.edu) and it, along with any other previous version, is superseded by this updated special publication version. Updates to this protocol may be released in the future. Visit http://nist.gov/mml/np-measurement-protocols.cfm to check for revisions of this protocol or new protocols in the series.

NIST and CEINT are interested in soliciting feedback on this method. We value user comments and suggestions to improve or further validate this protocol. Please send your name, email address and comments/suggestions to nanoprotocols@nist.gov. We also encourage users to report citations to published work in which this protocol has been applied. 


\section{Introduction}

Toxicity and fate assessment are key elements in the evaluation of the environmental, health and safety risks of engineered nanomaterials (ENMs). While significant effort and resources have been devoted to the toxicological evaluation of many ENMs, including nanoscale $\mathrm{TiO}_{2}$ [1-4], obtaining conclusive and reproducible results continues to be a challenge [5]. This can be traced in part to the lack of standardized dispersion protocols and the inconsistent application of dispersion procedures in relevant biological and environmental matrices $[6,7]$. In order to address these issues, the National Institute of Standards and Technology (NIST) jointly with the Center for the Environmental Implications of Nanotechnology (CEINT) have developed a series of standardized and validated protocols for the dispersion of ENMs from a powdered material source for both human health and environmental testing applications. This protocol has been developed and validated using NIST Standard Reference Material (SRM) 1898 . SRM 1898 consists of a widely studied and industrially relevant $\mathrm{TiO}_{2}$ nanomaterial with broad commercial penetration and a production history dating back several decades [3, 8-10].

While the procedures detailed in this series focus on the dispersion of SRM 1898 in specific aqueous media, it is believed that the adopted characterization, optimization and validation approaches can be more generally applied to the preparation of ENM dispersions in any relevant matrix. For this reason, and to allow for broader applicability, experimental details and discussions regarding the characterization, process optimization and validation steps adopted for the development of the dispersion method are detailed in a separate publication [11].

\section{Principles and scope}

In this protocol, $\mathrm{TiO}_{2}$ nanoparticle dispersions in relevant biological media are produced by following a series of steps applied to a stock $\mathrm{TiO}_{2}$ aqueous nanoparticle dispersion. To prepare the stock $\mathrm{TiO}_{2}$ aqueous dispersion, refer to the previous protocol in this series [12].

This protocol focuses on two representative media broadly used for in vitro assays: phosphatebuffered saline solution (PBS) and Dulbecco's Modified Eagle Medium containing $10 \%$ fetal bovine serum (DMEM-FBS). In both cases, bovine serum albumin (BSA) is utilized as a stabilizing agent; series specific serum albumin has been demonstrated to function as a nonspecific biocompatible stabilizer $[9,13,14]$. This protocol is proposed for the preparation of dispersions for generic acute bioassay applications; its use for chronic toxicity evaluations is beyond the scope of this work and should be validated by use of proper controls.

The method described herein, if applied correctly, yields $100 \mu \mathrm{g} / \mathrm{mL}$ monomodal nanoscale $\mathrm{TiO}_{2}$ dispersions in PBS or DMEM-FBS, characterized by mean particle diameters of $\approx 75 \mathrm{~nm}$ (in $\mathrm{PBS}$ ) and $\approx 83 \mathrm{~nm}$ (in DMEM-FBS) and $\mathrm{pH}$ values from 7.2 to 7.4 (in PBS) and $\approx 7.8$ (in DMEM-FBS). The dispersions retain their particle size distribution for $48 \mathrm{~h}$ under relevant incubation conditions (in DMEM-FBS) and at room temperature (in PBS). Dispersions prepared following this protocol should be stored so as to minimize exposure to light (e.g., in amber vials).

\footnotetext{
${ }^{a}$ Information regarding SRM 1898 can be accessed at http://www.nist.gov/srm/.
} 


\section{Terminology}

This protocol complies with definitions relevant to nanotechnology as set forth in the ASTM International standard E2456 [15] and is consistent with the draft standard ISO TS 80004-1 [16]. Additional guidance is derived from recommendations of the International Union of Pure and Applied Chemistry [17].

nanoparticle - sub-classification of ultrafine particle that is characterized by dimensions in the nanoscale (i.e., between approximately $1 \mathrm{~nm}$ and $100 \mathrm{~nm}$ ) in at least two dimensions; also referred to as "nano-object" in ISO TS 80004-1 [16].

primary particle - the smallest discrete identifiable entity associated with a particle system; in this context, larger particle structures (e.g., aggregates and agglomerates) may be composed of primary particles.

aggregate - a discrete assemblage of primary particles strongly bonded together (i.e., fused, sintered or metallically bonded).

Note-The adjective "primary," when used in conjunction with the term aggregate, is employed in the present context to indicate the smallest-achievable dispersed particle entity.

agglomerate - assemblage of particles (including primary particles and/or smaller aggregates) held together by relatively weak forces (e.g., van der Waals, capillary or electrostatic), that may break apart into smaller particles upon further processing.

Note-Although we define them as distinct entities, the terms aggregate and agglomerate have often been used interchangeably to denote particle assemblies.

dispersion - used in the present context to denote a liquid (aqueous) in which particles are homogeneously suspended, or the process of creating a suspension in which discrete particles are homogeneously distributed throughout a continuous fluid phase; implies the intention to break down agglomerates into their principal components (i.e., primary particles and/or aggregates).

\section{Reagents, materials and equipment}

\subsection{Reagents}

\subsection{1. $10 \mathrm{mg} / \mathrm{mL}$ stock $\mathrm{TiO}_{2}$ aqueous nanoparticle dispersion.}

Note-Refer to [12] for materials and guidelines for the preparation of the $10 \mathrm{mg} / \mathrm{mL}$ TiO2 aqueous dispersion.

4.1.2. Type I biological grade de-ionized (DI) water ( $\geq 18 \mathrm{M} \Omega \cdot \mathrm{cm}$ resistivity); biological grade implies sterility and absence of endotoxin contamination.

Note-Pyrogens (also known as endotoxins) are shed from the outer membrane of Gram-negative bacteria during cell division or lysis. These toxins are relatively heat-stable and are not destroyed under typical sterilizing conditions. As a result, pyrogens are ubiquitous and can interfere with the accuracy of toxicity assays. To depyrogenize glassware, bake at $250^{\circ} \mathrm{C}$ for $2 \mathrm{~h}$ or at $200^{\circ} \mathrm{C}$ overnight.

Note-Limulus Amoebocyte Lysate (LAL) reagent grade pyrogen-free water can be obtained from commercial vendors.

Note-Sterility and absence of pyrogen contamination should be verified for all materials in contact with the dispersion. If using the LAL test for pyrogens, avoid using cellulose-based filters, as they can be a source of beta-glucan, which interferes with the LAL assay. 
Note: If the dispersion is not intended for toxicological assessment, pyrogen-free conditions may not be necessary.

4.1.3. $0.1 \mathrm{~mol} / \mathrm{L} \mathrm{HCl}(\mathrm{aq})$ and $0.1 \mathrm{~mol} / \mathrm{L} \mathrm{NaOH}(\mathrm{aq})$ reagent grade solutions

4.1.4. Bovine serum albumin (BSA), 99+ \% high purity reagent grade (lipid and IgG free) powder (e.g., SeraCare Life Sciences, Code No. AP-4510-80)

\subsubsection{For dispersion in $P B S$ :}

PBS (10x) solution with $0.067 \mathrm{~mol} / \mathrm{L}\left(\mathrm{PO}_{4}\right)$, calcium and magnesium free, passed through $0.1 \mu \mathrm{m}$ sterile filter (e.g., HyClone Laboratories, Cat. No. SH30258.02)

\subsubsection{For dispersion in DMEM with $10 \%$ FBS:}

DMEM, $4.5 \mathrm{~g} / \mathrm{L}$ glucose and sodium pyruvate without L-glutamine \& phenol red, Sterile (e.g., Mediatech, Inc., Cat. No. 17-205-CV), with: Penicillin-Streptomycin (5000 U/mL Penicillin and $5000 \mathrm{ug} / \mathrm{mL}$ streptomycin), (e.g. Invitrogen Cat. No. 15070-063), Fungizone Antimycotic $(250 \mathrm{ug} / \mathrm{ml}$ amphotericin B and $205 \mathrm{ug} / \mathrm{mL}$ Sodium deoxycholate), (e.g. Invitrogen Cat No. 15290-018), and Glutimax Supplement (L-Alanyl- L-Glutamine, $200 \mathrm{mM}$ ), (e.g. Invitrogen, Cat. No. 35050-161)

The above preparation is referred to herein as DMEM

FBS (e.g., Gemini Bio-Products, Cat. No. 100-106)

\subsection{Materials}

4.2.1. $10 \mathrm{~mL}$ and $30 \mathrm{~mL}$ sterilized amber glass vials

4.2.2. Aluminum or polystyrene weighing dishes

4.3.2. Calibrated pipettes and sterile disposable tips covering a $(0.020$ to 5.000$) \mathrm{mL}$ range; adjustable volume pipettes are most convenient

\subsection{Equipment}

4.3.1. Analytical balance with readability to $0.1 \mathrm{mg}$

For verification of expected outcome:

4.3.3. $\mathrm{pH}$ meter

4.3.4. Laser Diffraction Spectrometer (LDS), or

4.3.5. Dynamic Light Scattering (DLS) instrument

\section{Preparation of $\mathrm{TiO}_{2}$ nanoparticle dispersions}

Note-To avoid contamination, all glassware in contact with the media or suspensions should be meticulously cleaned, rinsed with ethanol, and dried prior to use. Glassware can be sterilized using an autoclave, by exposure to hot, dry air $\left(130^{\circ} \mathrm{C}\right.$ to $\left.170^{\circ} \mathrm{C}\right)$ for $2 \mathrm{~h}$ to $4 \mathrm{~h}$ in an oven, or by prolonged contact with alcohol. Avoid detergents if possible; if detergents are used, rinse with copious amounts of DI water prior to rinsing with 
ethanol and drying. Store and work in high-efficiency particulate air (HEPA) filtered clean bench if available; if not, containers should be capped or sealed with thermoplastic (e.g., Parafilm).

Note-Use clean, sterile pipette tips and sterile procedures.

Note-For details on the validation of the particle size distribution (PSD) of dispersions, as well as the optimization of the BSA-TiO2 mixing sequence prescribed in this protocol, refer to [11].

\subsection{Dispersion in PBS}

5.1.1. Prepare a $10 \mathrm{mg} / \mathrm{mL}$ stock $\mathrm{TiO}_{2}$ aqueous nanoparticle dispersion, per [12].

5.1.2. Prepare $50 \mathrm{~mL}$ of PBS $(1 \mathrm{x})$ by doing a 1:10 dilution of the PBS (10x) with DI water, i.e., add $5 \mathrm{~mL}$ of PBS (10x) to $45 \mathrm{~mL}$ of DI water. Measure the $\mathrm{pH}$ of the prepared PBS (1x).

5.1.3. Adjust the $\mathrm{pH}$ of the prepared PBS (1x) solution to a value in the 7.2 to 7.4 range by addition of $0.1 \mathrm{~mol} / \mathrm{L} \mathrm{HCl}(\mathrm{aq})$ and/or $\mathrm{NaOH}(\mathrm{aq})$ as needed. Mix thoroughly after each acid or base addition step to allow for proper homogenization and attainment of equilibrium $\mathrm{pH}$.

Note-The $\mathrm{pH}$ range was selected as acceptable for typical biologically relevant assays. If other values are desired, the stability of the dispersions must be tested accordingly.

5.1.4. After addition of $\mathrm{HCl}(\mathrm{aq})$ and/or $\mathrm{NaOH}(\mathrm{aq})$ to achieve the desired $\mathrm{pH}$, add an adequate amount of PBS (10x) to compensate for the dilution of the PBS (1x) solution by the water added with the acid or base solutions; e.g., if $2 \mathrm{~mL}$ of $0.1 \mathrm{~mol} / \mathrm{L} \mathrm{HCl}$ (aq) were added to the PBS (1x) to obtain the desired $\mathrm{pH}$, then add $0.22 \mathrm{~mL}(2 \times 0.11)$ PBS (10x). After $\mathrm{pH}$ adjustment and equilibration, verify that the $\mathrm{pH}$ of the obtained PBS (1x) is in the range from 7.2 to 7.4 .

5.1.5. Weigh $0.8 \mathrm{~g}$ of BSA powder and transfer to a $10 \mathrm{~mL}$ amber glass vial. Add $10 \mathrm{~mL}$ of DI water to the vial with the BSA, seal and gently shake to allow for complete dissolution of the BSA while minimizing foam formation. Do not use the BSA solution until visible material is completely absent (allow approximately $1 \mathrm{~h}$ ). The final product is a transparent $80 \mathrm{mg} / \mathrm{mL}$ BSA solution in DI water.

5.1.6. Add $300 \mu \mathrm{L}$ of the $80 \mathrm{mg} / \mathrm{mL}$ aqueous BSA solution (5.1.5) into a clean $10 \mathrm{~mL}$ amber glass vial.

5.1.7. Add $150 \mu \mathrm{L}$ of the $10 \mathrm{mg} / \mathrm{mL}$ stock $\mathrm{TiO}_{2}$ aqueous dispersion (5.1.1) into the vial with $300 \mu \mathrm{L}$ of BSA solution (5.1.6).

5.1.8. In a $30 \mathrm{~mL}$ amber glass vial, add $14.5 \mathrm{~mL}$ of the PBS (1x) prepared in 5.1 .4 and $50 \mu \mathrm{L}$ of PBS (10x).

5.1.9. Using a calibrated pipette, transfer $450 \mu \mathrm{L}$ of the $\mathrm{TiO}_{2} / \mathrm{BSA} /$ water mixture obtained in (5.1.7) into the $30 \mathrm{~mL}$ vial with $14.55 \mathrm{~mL}$ of PBS solution (5.1.8), to yield a dispersion containing $100 \mu \mathrm{g} / \mathrm{mL} \mathrm{TiO}_{2}$ and $1.6 \mathrm{mg} / \mathrm{mL}$ BSA in PBS (1x). The $\mathrm{pH}$ of the resulting dispersion should be comparable to that of the original buffer medium (5.1.4).

5.1.10. For toxicological assays, the user is advised to conduct a control for BSA in the test medium $(1.6 \mathrm{mg} / \mathrm{mL})$ without $\mathrm{TiO}_{2}$.

5.1.11. The resulting dispersion, stored in the $30 \mathrm{~mL}$ amber vial, retains its PSD for at least $48 \mathrm{~h}$ at room temperature. 


\subsection{Dispersion in DMEM with $10 \%$ FBS}

5.2.1. Prepare a $10 \mathrm{mg} / \mathrm{mL} \mathrm{TiO}_{2}$ aqueous dispersion, per [12].

5.2.2. Prepare $50 \mathrm{~mL}$ of DMEM-FBS by mixing $5 \mathrm{~mL}$ of FBS with $45 \mathrm{~mL}$ of DMEM (1x). The resulting $\mathrm{pH}$ should be $\approx 7.8$.

5.2.3. Weigh $0.8 \mathrm{~g}$ of BSA powder and transfer to a $10 \mathrm{~mL}$ amber glass vial. Add $10 \mathrm{~mL}$ of DI water to the vial with the BSA, seal and gently shake to allow for complete dissolution of the BSA. Do not use the BSA solution until visible material is completely absent (allow approximately $1 \mathrm{~h}$ ). The final product is a transparent $80 \mathrm{mg} / \mathrm{mL}$ BSA solution in DI water.

5.2.4. Add $18.75 \mu \mathrm{L}$ of the $80 \mathrm{mg} / \mathrm{mL}$ aqueous BSA solution (5.2.3) into a clean $10 \mathrm{~mL}$ amber glass vial.

5.2.5. Add $150 \mu \mathrm{L}$ of the $10 \mathrm{mg} / \mathrm{mL} \mathrm{TiO}_{2}$ aqueous dispersion (5.2.1) into the vial with $18.75 \mu \mathrm{L}$ of BSA solution (5.2.4).

5.2.6. Using a calibrated pipette, add $14.83 \mathrm{~mL}$ of the DMEM-FBS prepared in 5.2 .2 to a $30 \mathrm{~mL}$ amber glass vial.

5.2.7. Transfer $168.75 \mu \mathrm{L}$ of the $\mathrm{TiO}_{2} / \mathrm{BSA} /$ water mixture obtained in (5.2.5) into the $30 \mathrm{~mL}$ vial with $14.83 \mathrm{~mL}$ of DMEM-FBS (5.2.6), to yield a $100 \mu \mathrm{g} / \mathrm{mL}\left(\mathrm{TiO}_{2}\right), 100 \mu \mathrm{g} / \mathrm{mL}(\mathrm{BSA})$ dispersion in DMEM-FBS. The $\mathrm{pH}$ of the resulting dispersion should still be comparable to that of the original medium (5.2.2). This procedure results in a $1.1 \%$ dilution of the DMEMFBS by the water added with the $\mathrm{TiO}_{2}$ and BSA stocks.

5.2.8. If used for toxicological assessments, the user is advised to conduct separate control tests for the BSA $(100 \mu \mathrm{g} / \mathrm{mL})$ in the medium and for the water-diluted medium $(1.1 \%)$, in the absence of $\mathrm{TiO}_{2}$.

5.2.9. The resulting dispersion, stored in the $30 \mathrm{~mL}$ amber vial, retains its $\mathrm{PSD}$ and $\mathrm{pH}( \pm 0.1$ units) for at least $48 \mathrm{~h}$ under relevant incubation conditions $\left(37^{\circ} \mathrm{C}, 90 \%\right.$ humidity, $5 \% \mathrm{CO}_{2}$ atmosphere).

\section{Expected outcome}

Note-The particle size distribution (PSD) of the resulting dispersions was monitored for $48 \mathrm{~h}$, corresponding to the typical acute toxicity assay timeframe. Although beyond the scope of this protocol, dispersions may remain stable for longer periods of time. This behavior has been validated only in pure media, without the presence of cells or other added components.

Note-Serial dilutions may cause agglomeration. This should be tested accordingly.

\subsection{Dispersion in PBS}

6.1.1. The obtained dispersions should have a white but translucent appearance if prepared using SRM 1898 or commercial P25.

Note-If source powders other than P25 are used, the appearance may vary depending on the final particle size, particle concentration and other factors. 
6.1.2. The particle size distribution (PSD) of the dispersion should be monomodal, with the following volume-based mean particle diameter $\left(D_{m}\right), D_{10}$ and $D_{90}$ values: ${ }^{b}$

If measured using LDS:

$$
\begin{aligned}
& \mathrm{D}_{\mathrm{m}} \approx(71 \text { to } 79) \mathrm{nm} \\
& \mathrm{D}_{10} \approx(60 \text { to } 64) \mathrm{nm} \\
& \mathrm{D}_{90} \approx(80 \text { to } 96) \mathrm{nm}
\end{aligned}
$$

If measured using DLS:

$$
\begin{aligned}
& \mathrm{D}_{\mathrm{m}} \approx(122 \text { to } 149) \mathrm{nm} \\
& \mathrm{D}_{10} \approx(69 \text { to } 91) \mathrm{nm} \\
& \mathrm{D}_{90} \approx(160 \text { to } 200) \mathrm{nm}
\end{aligned}
$$

The expected size range was calculated from three independent replicates obtained following the prescribed procedure. Refer to the Appendix for details on the calculation of the expected size parameter ranges.

The volume-based mean particle diameter, as well as the $\mathrm{D}_{10}$ and $\mathrm{D}_{90}$ values, for aqueous $\mathrm{P} 25$ dispersions prepared following the protocol should be reported by the user to allow for comparison with the values specified herein. Refer to [18] for details on PSD characterization and validation criteria, as well as representative PSD profiles.

6.1.3 The $\mathrm{pH}$ of dispersions should be comparable $( \pm 0.1 \mathrm{pH}$ units $)$ to the $\mathrm{pH}$ of the originating medium.

\subsection{Dispersion in DMEM with $10 \%$ FBS}

6.2.1. The obtained dispersions should have a white but translucent appearance if prepared using SRM 1898 or commercial P25.

Note - If source powders other than P25 are used, the appearance may vary depending on the final particle size, particle concentration and other factors.

6.2.2. The particle size distribution (PSD) of the dispersion should be monomodal, with the following volume-based mean particle diameter, $\left(\mathrm{D}_{\mathrm{m}}\right), \mathrm{D}_{10}$ and $\mathrm{D}_{90}$ values:

If measured using LDS:

$$
\begin{aligned}
& \mathrm{D}_{\mathrm{m}} \approx(81 \text { to } 85) \mathrm{nm} \\
& \mathrm{D}_{10} \approx(61 \text { to } 69) \mathrm{nm} \\
& \mathrm{D}_{90} \approx(94 \text { to } 115) \mathrm{nm}
\end{aligned}
$$

If measured using DLS:

$$
\mathrm{D}_{\mathrm{m}} \approx(138 \text { to } 170) \mathrm{nm}
$$

\footnotetext{
${ }^{\mathrm{b}} \mathrm{D}_{10}$ and $\mathrm{D}_{90}$ refer to characteristic percentile size values associated with the cumulative volume or mass less than $10 \%$ and $90 \%$, respectively, of the total volume or mass within the distribution. These parameters are routinely reported by LDS instruments. They may or may not be obtainable directly from commercial DLS instruments, depending on the manufacturer.
} 


$$
\begin{aligned}
& \mathrm{D}_{10} \approx(86 \text { to } 113) \mathrm{nm} \\
& \mathrm{D}_{90} \approx(162 \text { to } 337) \mathrm{nm}
\end{aligned}
$$

The expected range for size parameters was calculated from three independent replicates obtained following the prescribed procedure. Refer to the Appendix for details on the calculation of the expected size parameter ranges.

The volume-based mean particle diameter, as well as the $\mathrm{D}_{10}$ and $\mathrm{D}_{90}$ values, for aqueous P25 dispersions prepared following the protocol, should be reported by the user to allow for comparison with the values specified herein. Refer to [18] for details and discussions on PSD characterization and validation criteria, as well as illustrations of representative PSD profiles.

6.2.3. The $\mathrm{pH}$ of dispersions should be comparable $( \pm 0.1 \mathrm{pH}$ units) to the $\mathrm{pH}$ of the originating medium.

\section{Abbreviations}

BSA bovine serum albumin

DI de-ionized

DLS dynamic light scattering

DMEM-FBS Dulbecco's Modified Eagle Medium containing 10 \% FBS

ENM engineered nanomaterial

FBS fetal bovine serum

HEPA high-efficiency particulate air

ISO International Organization for Standardization

IUPAC International Union of Pure and Applied Chemistry

LDS laser diffraction spectrometry

PBS phosphate buffered saline

PSD particle size distribution

SRM Standard Reference Material (a registered trademark of the National Institute of Standards and Technology)

\section{Acknowledgements}

We thank John Elliott, NIST Biochemical Science Division, for providing access to an incubator and for providing the DMEM-FBS test media used in the development of this protocol.

\section{References}

1. Landsiedel, R., et al., Testing metal-oxide nanomaterials for human safety. $J . A d v$. Mater., 2010. 22(24): p. 2601-2627. 
2. Pichat, P., A brief survey of the potential health risks of $\mathrm{TiO} 2$ particles and $\mathrm{TiO} 2-$ containing photocatalytic or non-photocatalytic materials. J. Adv. Oxid. Technol., 2010. 13(3): p. 238-246.

3. Warheit, D.B., et al., Pulmonary toxicity study in rats with three forms of ultrafine-TiO2 particles: Differential responses related to surface properties. Toxicology, 2007. 230(1): p. 90-104.

4. $\quad \mathrm{Wu}, \mathrm{J} . \mathrm{H}$., et al., Toxicity and penetration of $\mathrm{TiO} 2$ nanoparticles in hairless mice and porcine skin after subchronic dermal exposure. Toxicology Lett., 2009. 191(1): p. 1-8.

5. Titanium Dioxide (TiO2) IARC Monograph 93. International Agency for Research on Cancer, 2006.

6. Roebben, G., et al., Interlaboratory reproducibility of size and surface charge measurements on nanoparticles prior to biological impact assessment. Nature Nanotech., 2010. 13(7): p. 2675-2687.

7. Taurozzi, J.S., V.A. Hackley, and M.R. Wiesner, Ultrasonic dispersion of nanoparticles for environmental, health and safety assessment - Issues and recommendations. Nanotoxicology, 2011. 5(4): p. 711-729.

8. Standard Reference Material 1898 (in production), National Institute of Standards and Technology, Gaithersburg, MD, http://www.nist.gov/srm/.

9. List of Manufactured Nanomaterials and List of Endpoints for Phase One of the OECD's Testing Program, Series on the safety of manufactured nanomaterials, Number 6, 2008, Environmental Diractorate, Organization for Economic Co-operation and Development. Paris, France.

10. Porter, D., et al., A biocompatible medium for nanoparticle dispersion. Nanotoxicology, 2008. 2(3): p. 144-154.

11. Taurozzi, J.S., V.A. Hackley, and M.R. Wiesner, A standardized approach for the dispersion of titanium dioxide nanoparticles in biological media. Nanotoxicology, 2011. Submitted.

12. Taurozzi, J.S., V.A. Hackley, and M.R. Wiesner, Preparation of a nanoscale TiO2 aqueous dispersion for toxicological or environmental testing. NIST SP 1200-3, National Institute of Standards and Technology, Gaithersburg, MD, June 2012.

13. MacCuspie, R.I., A.J. Allen, and V.A. Hackley, Dispersion stabilization of silver nanoparticles in synthetic lung fluid studied under in-situ conditions. Nanotoxicology, 2010. Early Online: p. 1-17.

14. Tsai, D.H., et al., Adsorption and conformation of serum albumin protein on gold nanoparticles investigated using dimensional measurements and in situ spectroscopic methods. Langmuir, 2011. 27(6): p. 2464-2477.

15. E2456-06: Standard terminology relating to nanotechnology. 2006, ASTM International

16. ISO TS 80004-1: Nanotechnologies - Vocabulary - Part 1: Core terms. 2007, International Standards Organization, Geneva, Switzerland.

17. Version 2009-09-07, Release 2.1.5: Compendium of chemical terminology. 2009, International Union of Pure and Applied Chemistry (IUPAC).

18. Taurozzi, J.S., V.A. Hackley, and M.R. Wiesner, A standardized approach for the dispersion of titanium dioxide nanoparticles in biological media. Nanotoxicology, 2011. submitted. 


\section{Appendix}

\section{A.1. Calculation of expected particle size parameters}

The expected range for $\mathrm{D}_{\mathrm{m}}, \mathrm{D}_{10}$ and $\mathrm{D}_{90}$ values was obtained using the following equation:

$$
\text { range }=\left(x-\frac{t . s}{\sqrt{n}} \text { to } x+\frac{t . s}{\sqrt{n}}\right)
$$

Where $\boldsymbol{x}$ and $\boldsymbol{s}$ are the average and standard deviation, respectively, of the measured size parameter from three independent replicates, $t$ is the student test parameter for a $95 \%$ confidence interval and two degrees of freedom $(\boldsymbol{t}=4.30)$, and $\boldsymbol{n}$ is the number of tested samples $(\boldsymbol{n}=3)$. 